\title{
A Tale of Two Sites: On Defining the Carrier Concentration in Garnet-Based Ionic Conductors for Advanced Li Batteries
}

\author{
Travis Thompson, Asma Sharafi, Michelle D. Johannes, Ashfia Huq, Jan L. Allen, \\ Jeff Wolfenstine, and Jeff Sakamoto*
}

Solid electrolytes based on the garnet crystal structure have recently been identified as a promising material to enable advance Li battery cell chemistries because of the unprecedented combination of high ionic conductivity and electrochemical stability against metallic Li. To better understand the mechanisms that give rise to high conductivity, the goal of this work is to correlate Li site occupancy with Li-ion transport. Toward this goal, the Li site occupancy is studied in cubic garnet as a function of Li concentration over the compositions range: $\mathrm{Li}_{7-x} \mathrm{La}_{3} \mathrm{Zr}_{2-x} \mathrm{Ta}_{x} \mathrm{O}_{12}(x=0.5,0.75$, and 1.5). The distribution of Li between the two interstitial sites ( $24 \mathrm{~d}$ and $96 \mathrm{~h}$ ) is determined using neutron and synchrotron diffraction. The bulk conductivity is measured on $>\mathbf{9 7 \%}$ relative density polycrystalline specimens to correlate Li-ion transport as a function of Li site occupancy. It is determined that the conductivity changes nonlinearly with the occupancy of the octahedral (96h) Li site. It is shown that the effective carrier concentration is dependent on the Li site occupancy and suggests that this is a consequence of significant carriercarrier coulombic interactions. Furthermore, the observation of maximum conductivity near $\mathrm{Li}=6.5 \mathrm{~mol}$ is explained.

\section{Introduction}

Li-ion battery technology has advanced significantly in the last two decades: $;{ }^{[1-4]}$ however, future energy storage demands will require safer, cheaper, and higher performance electrochemical

Dr. T. Thompson, A. Sharafi, Prof. J. Sakamoto

Department of Mechanical Engineering

University of Michigan

2350 Hayward Ave, GG Brown Laboratory

AnnA rbor, MI 48109, USA

E-mail: jeffsaka@umich.edu

Dr. M. D. Johannes

Center for Computational Materials Science

Naval Research Laboratory

Anacostia, VA 20375, USA

Dr. A. Huq

Spallation Neutron Source

Bear Creek Rd

Oak Ridge National Laboratory

OakRid ge, TN 37831,USA

Dr. J. L. Allen, Dr. J. Wolfenstine

RDRL-SED-C

Army Research Laboratory

2800Po wderM illRo ad

Adelphi, MD 20783, USA

DOI: 10.1002/aenm.201500096 energy storage. While the primary strategy for improving performance has focused on electrode materials, the development of new solid state ionic conducting electrolytes has been overlooked as a potential means to revolutionize electrochemical energy storage. Examples of some of the technologies (Figure 1) could include: (i) dual electrolyte Li-S batteries, (ii) solidstate batteries employing Li metal anodes, and (iii) all oxide, solid-state Li-ion batteries. Indeed, the need for ionic conducting solid-state electrolytes is clear, but relatively few have been identified as promising candidates. The garnet mineral structure represents a family of complex oxides spanning a broad range of natural and synthetic compositions.

Initial work identified $\mathrm{Li}_{5} \mathrm{La}_{3} \mathrm{M}_{2} \mathrm{O}_{12}$ $\left(\mathrm{M}=\mathrm{Nb}^{5+}, \mathrm{Ta}^{5+}\right)$ as a promising formulation having a total conductivity of $0.04 \mathrm{mS} \mathrm{cm}{ }^{-1}$ at room temperature. ${ }^{[5-7]}$ Recently, Weppner and co-workers discovered a formulation exhibiting up to $0.4 \mathrm{mS} \mathrm{cm}^{-1}$ at room temperature: $\mathrm{Li}_{7} \mathrm{La}_{3} \mathrm{Zr}_{2} \mathrm{O}_{12}$ (also known as LLZO). ${ }^{[8]}$ For comparison, conventional battery separators soaked in liquid electrolyte have a total conductivity of $0.4 \mathrm{mS} \mathrm{cm}^{-1}$ at room temperature. ${ }^{[9]}$ In LLZO, edge-sharing $\mathrm{ZrO}_{6}$ octahedra and $\mathrm{LaO}_{8}$ dodecahedra form an isotropic skeleton framework through which Li-ions and Li-ion vacancies form a percolative network of tetrahedral and distorted octahedral sites (Figure 1). In addition to high conductivity, LLZO has been reported to have the unprecedented combination of stability against metallic $\mathrm{Li}$, and stability in dry air. ${ }^{[8,10-12]}$

LLZO is also referred to as a "stuffed" garnet because of its relatively high $\mathrm{Li}$ concentration $(7$ moles $)$ compared to $\mathrm{Li}_{3} \mathrm{La}_{3} \mathrm{M}_{2} \mathrm{O}_{12}$. In the garnet family of materials, as the Li content is increased from $\mathrm{Li}=3$ mols toward $\mathrm{Li}=7$ mols, the ionic conductivity increases by several orders of magnitude. ${ }^{[13]} \mathrm{At} \mathrm{Li}$ contents near $\mathrm{Li}=6.5 \mathrm{mols}$, the cubic structure undergoes a reduction of symmetry to a tetragonal polymorph and the ionic conductivity again decreases. ${ }^{[14,15]} \mathrm{A}$ maximum in the ionic conductivity at room temperature has been observed for compositions near the critical Li concentration to stabilize the cubic phase. ${ }^{[16-19]}$ As such, the majority of the work in the literature has focused on understanding how the cubic phase is stabilized at high Li concentrations. ${ }^{[14-21]}$ However, the understanding of the fundamental parameters that control the Li conductivity has 


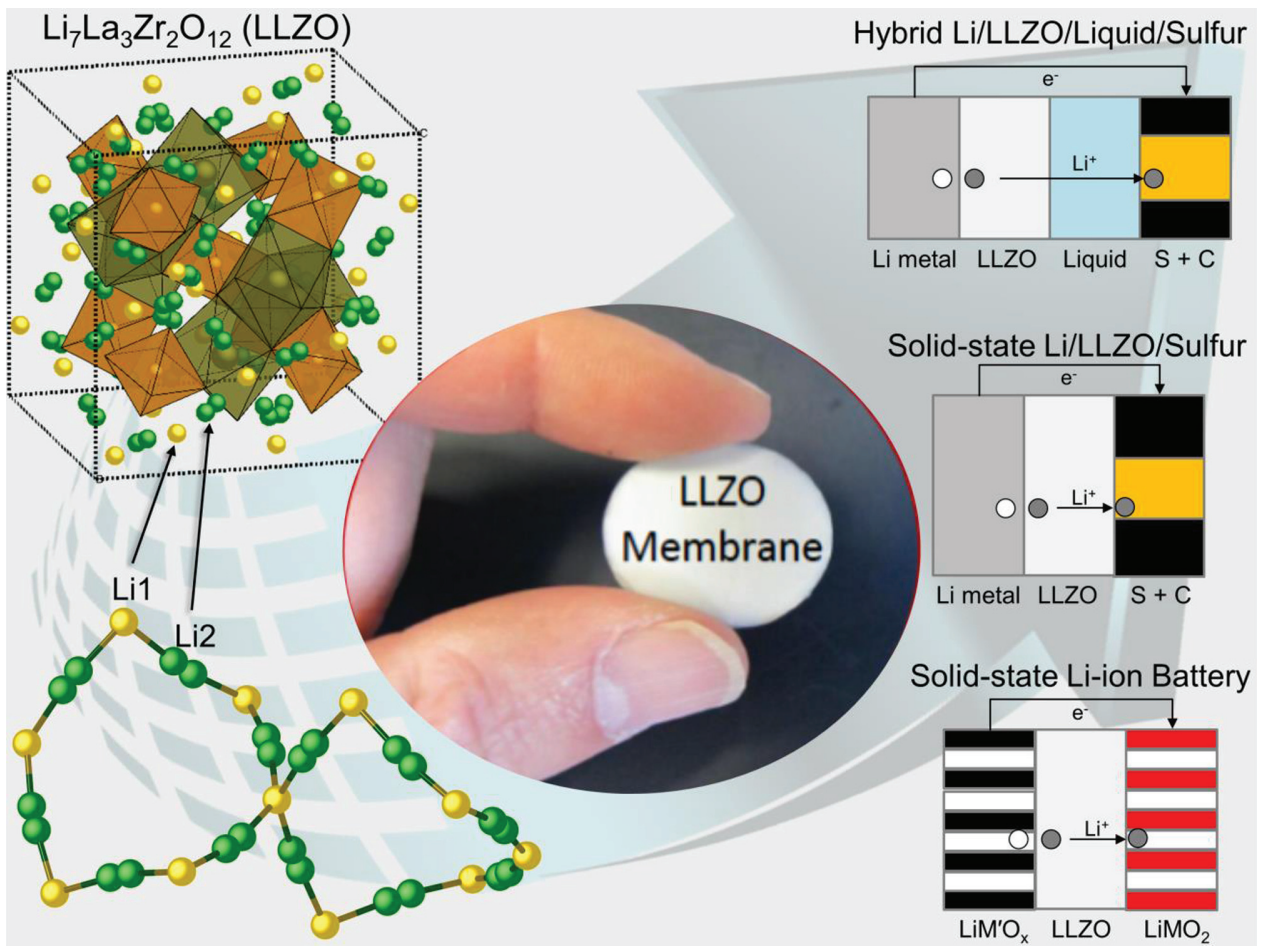

Figure 1. Schematic showing the cubic crystal structure, the two Li sites, and possible configurations of devices that utilize LLZO.

been limited and the mechanism that leads to high ionic conductivity requires elucidation.

Ionic conductivity follows the relationship: $\sigma=\mu q \eta$, where $\mu$ is the carrier mobility, $q$ is the carrier valence, and $\eta$ is the carrier concentration. The conductivity is governed by the carrier concentration and the mobility, since the charge is fixed at 1 for Li-ion conductors. Thus, in order to understand the Li-ion conductivity, the carrier mobility and concentration must be well defined and the variables that affect these parameters must be understood. As will be shown in this work, one such variable that has not been fully explored is the site occupancy on each of the two Li sites in the garnet crystal structure. We will show that the occupancy of the two Li sites (see Figure 1 for a graphical description of the two Li sites) plays a crucial role in how the carrier concentration is defined and explains the peak observed in the conductivity.

Models have been developed for single site conductors with no carrier-carrier interaction to mathematically explain the charge transport. ${ }^{[22],[N e r n s t-E i n s t e i n ~ r e l a t i o n s h i p] ~ I n ~ t h e s e ~ t h e o r e t i c a l ~}$ models, the carrier concentration is equivalent to the Li content, i.e., all the ions participate in conduction. This assumption was contested previously in the literature for other families of ionic conductors. ${ }^{[23-27]}$ Subsequently, ionic Hall effect measurements were performed and it was found that the Hall mobility only agreed with the drift mobility if all the ions were participating in conduction. ${ }^{[23,28]} \mathrm{Li}$ conducting garnets have been analyzed using the same assumption that all the Li-ions contribute to the conductivity. ${ }^{[10,16,19]}$ However, recent experimental evidence shows that this assumption is not valid for the garnet family of materials. ${ }^{[29]}$ Thus, in order to clarify what is the effective carrier concentration in the garnet family of ionic conductors, a compositional series of cubic stabilized garnet ionic Li ion conductors, $\mathrm{Li}_{7-x} \mathrm{La}_{3} \mathrm{Zr}_{2-x} \mathrm{Ta}_{x} \mathrm{O}_{12}(\mathrm{x}=0.5,0.75$, and 1.5), was synthesized and the relationship between site occupancy and effective carrier concentration was determined.

We hypothesize that stabilizing the higher conducting cubic LLZO by substituting $\mathrm{Ta}^{5+}$ for $\mathrm{Zr}^{4+}$ is preferred relative to $\mathrm{Al}^{3+}$ for $\mathrm{Li}^{+}$since $\mathrm{Ta}$, unlike $\mathrm{Al}$, does not reside on the $\mathrm{Li}$ sublattice. ${ }^{[30-32]}$ This substitution method facilitates a correlation between Li-ion site occupancy and bulk conductivity in LLZO. In this study, the degree of substitution was varied over a broad range to help establish trends in Li site occupancy/concentration and conductivity; $\mathrm{Li}_{7-x} \mathrm{La}_{3} \mathrm{Zr}_{2-x} \mathrm{Ta}_{x} \mathrm{O}_{12}(x=0.5,0.75$, and 1.5). A composition with the formula $\mathrm{Li}_{7-x} \mathrm{La}_{3} \mathrm{Zr}_{2-x} \mathrm{Ta}_{x} \mathrm{O}_{12}$ $(x=0.25)$ was also investigated by us recently and found to be a mixture of both the tetragonal and cubic phases. ${ }^{[15]}$ Since the purpose of this study is to investigate cubic garnets, that composition was omitted from this study.

A hot pressing technique was employed to rapidly heat LLZO powders while applying pressure to minimize grain boundary impedance so that the bulk response could be isolated and maximize density without depending on $\mathrm{Al}$ to enhance sintering. The hot pressing technique achieved uniformly high relative densities $(\geq 97 \%)$ to allow for the accurate measurement of the bulk and grain boundary conductivities. By accurately measuring the bulk conductivity, based on the $\mathrm{Zr} / \mathrm{Ta}$ ratio, the relationship between Li site occupancy and ionic transport can be elucidated. The purpose of this work was to conduct a detailed study to understand the variables (such as Li site occupancy) involved in maximizing bulk conductivity in cubic LLZO based garnets with the compositional series $\mathrm{Li}_{7-x} \mathrm{La}_{3} \mathrm{Zr}_{2-x} \mathrm{Ta}_{x} \mathrm{O}_{12}(x=$ $0.5,0.75$, and 1.5 ) and to begin discussion on how the carrier concentration should be defined. The experimental results from this work were compared to the results in the literature, 


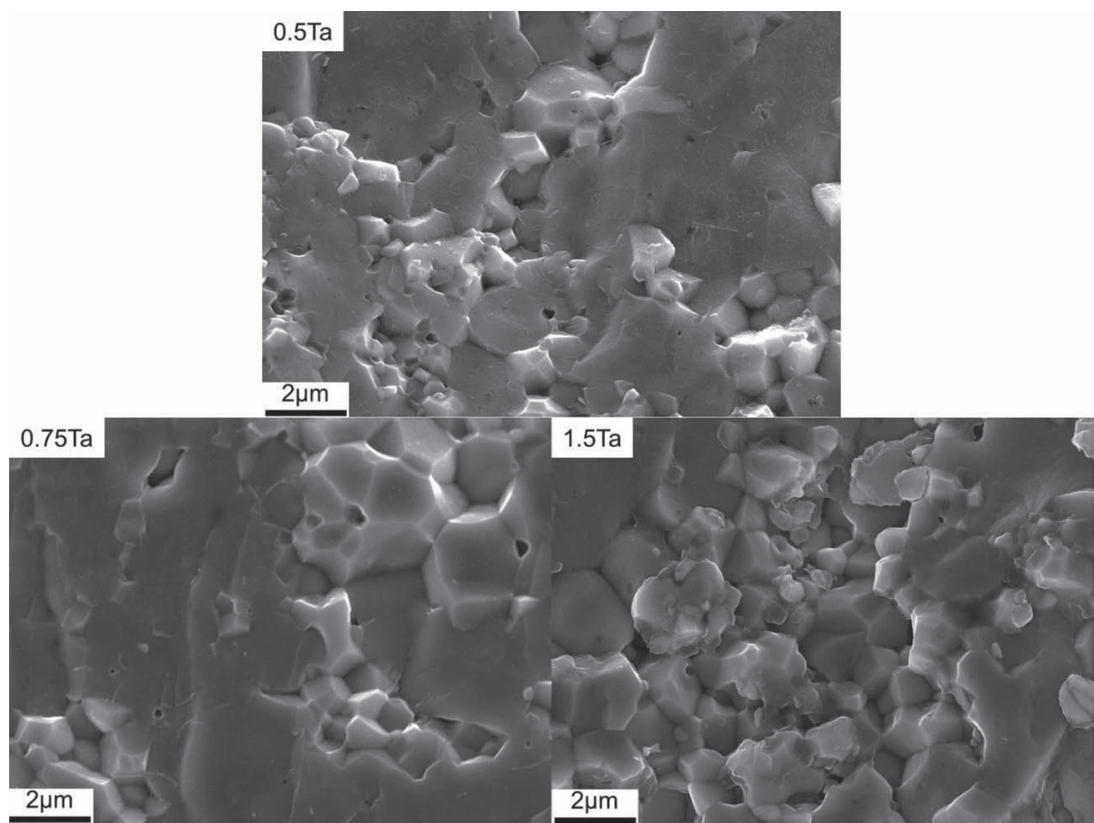

Figure 2. SEM images of fracture surfaces of each composition in the series $\mathrm{Li}_{7-x} \mathrm{La}_{3} \mathrm{Zr}_{2-x} \mathrm{Ta}_{x} \mathrm{O}_{12}(x=0.5,0.75$, and 1.5).

when available. This is the first report of the occupancy of two Li sites (96h and 24d) over a broad compositional range without the presence of Al. This work presents compelling evidence to better understand the relationship between the ionic conductivity, mobility, and carrier concentration in solid electrolytes based on the garnet crystal structure.

\section{Results and Discussion}

After hot pressing the powders, scanning electron microscopy (SEM) analysis was performed on fracture surfaces to characterize the fracture mode, grain size, and porosity. Representative microstructures for each composition in the series $\mathrm{Li}_{7-x} \mathrm{La}_{3} \mathrm{Zr}_{2-x} \mathrm{Ta}_{x} \mathrm{O}_{12}(x=0.5,0.75$, and 1.5) are shown in Figure 2. All samples had relatively dense microstructures with grain sizes between 1.2 and $1.4 \mu \mathrm{m}$ (Table 1) and exhib-

Table 1. Summary of microstructural and transport data extracted from the SEM, EIS, and Rietveld analysis.

\begin{tabular}{lccc}
\hline & \multicolumn{3}{c}{ Nominal Ta content [mol] } \\
\cline { 2 - 4 } & 0.5 & 0.75 & 1.5 \\
\hline RT total $\sigma\left[\mathrm{mS} \mathrm{cm}^{-1}\right]$ & 0.816 & 0.390 & 0.080 \\
RT bulk $\sigma\left[\mathrm{mS} \mathrm{cm}^{-1}\right]$ & 0.903 & 0.405 & 0.103 \\
Total Ea $[\mathrm{eV}]$ & 0.431 & 0.452 & 0.561 \\
Bulk Ea $[\mathrm{eV}]$ & 0.435 & 0.443 & 0.536 \\
Grain size $[\mu \mathrm{m}]$ & 1.4 & 1.3 & 1.2 \\
Relative density & $97.8 \%$ & $97.1 \%$ & $97.3 \%$ \\
Lattice parameter [尺̊] & 12.93 & 12.91 & 12.84 \\
24d-96h Li-Li separation distance $[\AA ̊]$ & 1.61 & 1.62 & 1.64 \\
96h-96h Li-Li separation distance $[\AA ̊]$ & 0.77 & 0.75 & 0.68 \\
\hline
\end{tabular}

ited a mixed inter- and transgranular fracture mode similar to previous reports of LLZO.[21] The theoretical density was calculated using the diffraction data and had relative densities $\geq 97 \%$. The high relative densities, similar grain sizes, and fracture mode indicate that Ta-doped LLZO samples without $\mathrm{Al}$ can be consolidated close to full theoretical density without the use of a sintering aid and that all the samples in this study had similar microstructures. Furthermore, because the microstructural features were similar among the samples, comparison of the bulk transport properties between compositions is possible.

Electrochemical impedance spectroscopy (EIS) was performed as a function of temperature. For this work, the EIS data were modeled using a modified equivalent circuit proposed by Huggins. ${ }^{[33]}$ This model was chosen since it better represents the physical arrangement of the cell hardware used to perform the EIS compared to the conventional brick-layer model. For a full development of the equivalent circuit modeling used here, the reader is directed to the Supporting Information. Figure 3 shows a representative Nyquist plot of the EIS data for composition $\mathrm{Li}_{6.5} \mathrm{La}_{3} \mathrm{Zr}_{1.5} \mathrm{Ta}_{0.5} \mathrm{O}_{12}$ at room temperature, the equivalent circuit and the modeled impedance response. The salient features are labeled with their characteristic frequencies and semicircles are included to help guide the eye.

An Arrhenius plot of the bulk conductivities was extracted from the impedance spectra by the equivalent circuit modeling and is included in Figure S1, Supporting Information. Table 1 summarizes the conductivity and activation energy values from the Nyquist and Arrhenius data, respectively. Based on the data in Table 1, several observations can be made. First, for each composition, the bulk and total conductivities are similar and the bulk conductivity decreases with increasing Ta content. The low grain boundary resistance is expected because of the high relative density resulting from the hot-pressing technique used, as was previously demonstrated. ${ }^{[15]}$ The resulting presence of significant transgranular fracture observed in the SEM images indicates good grain-to-grain adhesion (Figure 2). Second, the activation energies of $>0.4 \mathrm{eV}$ observed are higher than what has been reported for samples that contain Ta and Al. ${ }^{[16,32]}$ However, in recent Ta-stabilized LLZO series made without Al, the activation energies agree well. ${ }^{[17]}$ It has been demonstrated that LLZO samples stabilized with only Al have low activation energies on the order of $0.26-0.33 \mathrm{eV}^{[8,12,21]}$ It is possible that the $\mathrm{Al}$ in these Ta-stabilized LLZO literature references is lowering the activation energy. How the presence of $\mathrm{Al}$ lowers the activation energy is unclear at this time.

Synchrotron X-ray diffraction and neutron diffraction were performed on the series $\mathrm{Li}_{7-x} \mathrm{La}_{3} \mathrm{Zr}_{2-x} \mathrm{Ta}_{x} \mathrm{O}_{12}(x=0.5,0.75$, and 1.5). A typical neutron diffraction data refinement after convergence is shown in Figure 4A. For brevity, only the $x=0.5 \mathrm{Ta}$ diffraction pattern (the sample with the highest ionic conductivity) is shown in this report and the remaining diffraction patterns are included in Figures S2-S12, Supporting Information. 


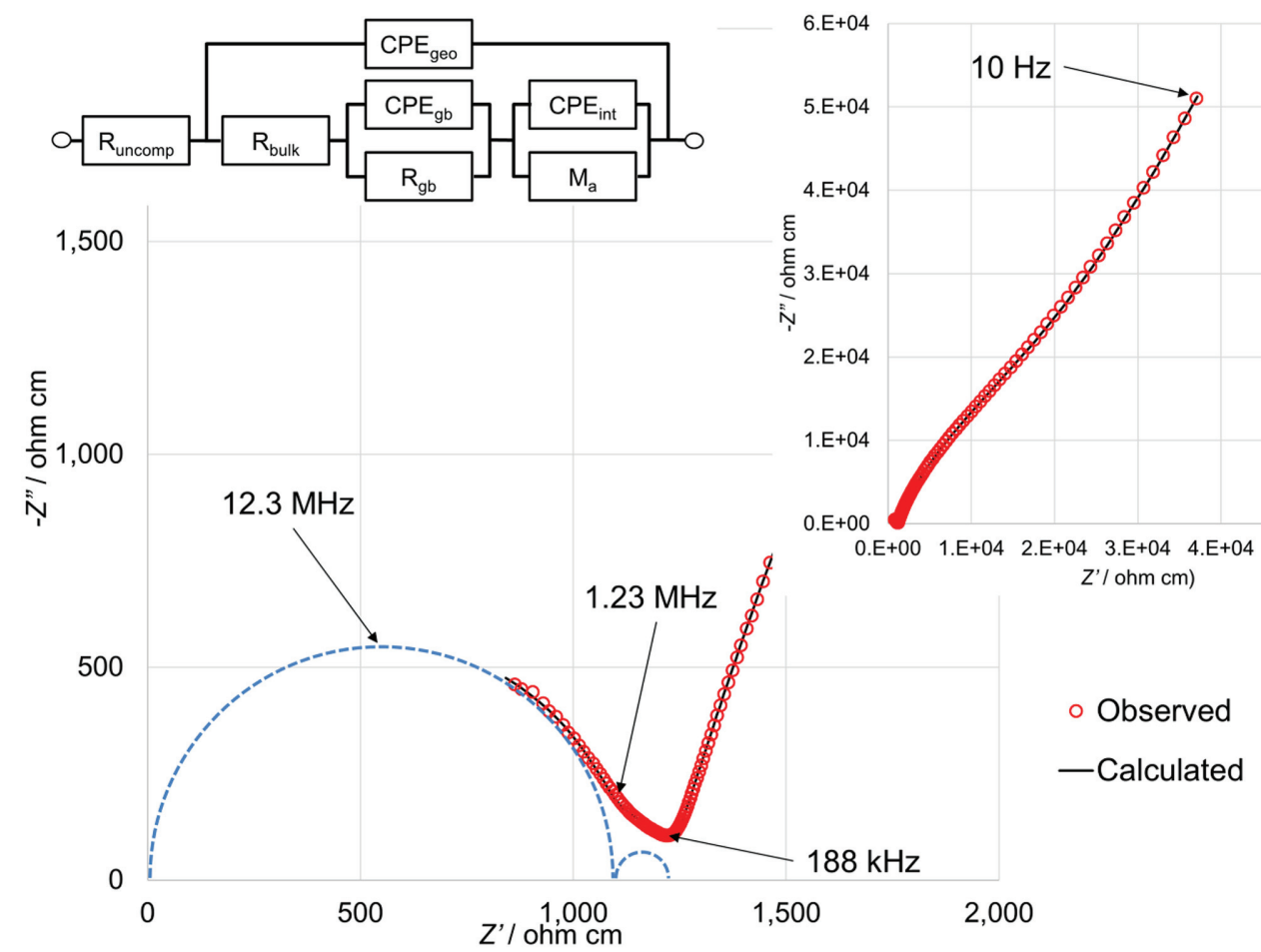

Figure 3. Ionic transport data obtained from electrochemical impedance spectroscopy in Ta-doped LLZO. Representative Nyquist plot (for $x=0.5$ Ta), equivalent circuit used to model the data, and the calculated impedance response. The salient features are labeled with their characteristic frequencies and semicircles are included to help guide the eye.

Since X-ray diffraction is not sensitive to light elements such as Li with low Z numbers and Ta and Zr have similar neutron scattering cross-sections, both synchrotron X-ray and neutron diffraction were performed. This complementary approach allowed for accurate structural refinement (Table 2, Tables S1 and S2, Supporting Information).

Figure $4 \mathrm{~B}$ plots the lattice parameter versus nominal Ta content constructed from the structural refinement data and relevant literature values. The literature references included in Figure 4B correspond to other $\mathrm{Zr} / \mathrm{Ta}$ garnet solid solutions of similar compositions. ${ }^{[16,34-36]}$ The lattice parameters of this work agree well with the values from the literature. As expected, the $\mathrm{Zr} / \mathrm{Ta}$ solid solution is following Vegard's Law where the lattice parameter is linearly changing with increasing substitution. Furthermore, the lattice parameter decreases with increased Ta substitution, which is expected since the $\mathrm{Ta}^{5+}$ ion in sixfold coordination is smaller than the $\mathrm{Zr}^{4+}$ with the same coordination (78 and $86 \mathrm{pm}$, respectively). ${ }^{[37]}$

The Li site occupancies have been refined for this compositional series from the diffraction data and are summarized in Figure 5A,B. The results were compared to literature values for similar Li contents and for garnets based on other formulations with significantly lower Li contents. For a full description of the structural model used, the reader is referred to the Supporting Information. The linear fits to the data of Cussen et al. and O'Callaghan et al. are for Li garnets of a variety of formulations based on W, Sb, and Ta with lower Li contents. ${ }^{[38-42]}$ The data sets of Logéat et al. ${ }^{[34]}$ and Goodenough and co-workers ${ }^{[16]}$ are for $\mathrm{Zr} / \mathrm{Ta}$ solid solutions similar in composition to the series explored in this work. For the data of this work, uncertainties were calculated but were smaller than the symbol and have been omitted.

Accordingly, the general trend of the Li occupancy in the two coordination environments for our experimental series are in excellent agreement with reported literature values for garnets not only stabilized by $\mathrm{Ta}$ with $\mathrm{Al}$ present but also for the garnets of significantly different formulations investigated by Cussen et al. and $\mathrm{O}^{\prime}$ Callaghan et al. ${ }^{[38-42]}$ As the Li content is increased, the tetrahedrally coordinated (24d) Li sites depopulate and the octahedrally coordinated (96h) Li sites populate. This is significant because the $96 \mathrm{~h}$ site is not normally populated in the garnet structure, ${ }^{[43]}$ yet the trend is obvious over a wide compositional range. The increase of $\mathrm{Li}$ in the $96 \mathrm{~h}$ site is important since NMR reports show that the Li ions occupying the $96 \mathrm{~h}$ site are more mobile than those occupying the $24 \mathrm{~d}$ site. ${ }^{[4-47]}$ In addition, Goodenough and co-workers suggest that a $3: 1$ ratio of $96 \mathrm{~h} / 24 \mathrm{~d}$ population is the optimum for high ionic conductivity. ${ }^{[16]}$ How the site occupancy is correlated to the bulk conductivity is discussed next.

For the garnet system, where the Li content is changed by the introduction of a dopant, it has been empirically observed in the literature that the ionic conductivity at room temperature passes through a maximum near a nominal Li content of 6.5 mols. ${ }^{[16-19]}$ The prevailing assumption has been that the Li content (i.e., carrier concentration) is responsible for the high Li-ion conductivity. ${ }^{[10,16,19]}$ However, Ohta et al. noted that when the carrier concentration is calculated based on this assumption, it can be found that the conductivity is not increasing linearly with the carrier concentration. Since the carrier concentration (assuming all the ions participate in conduction) does 

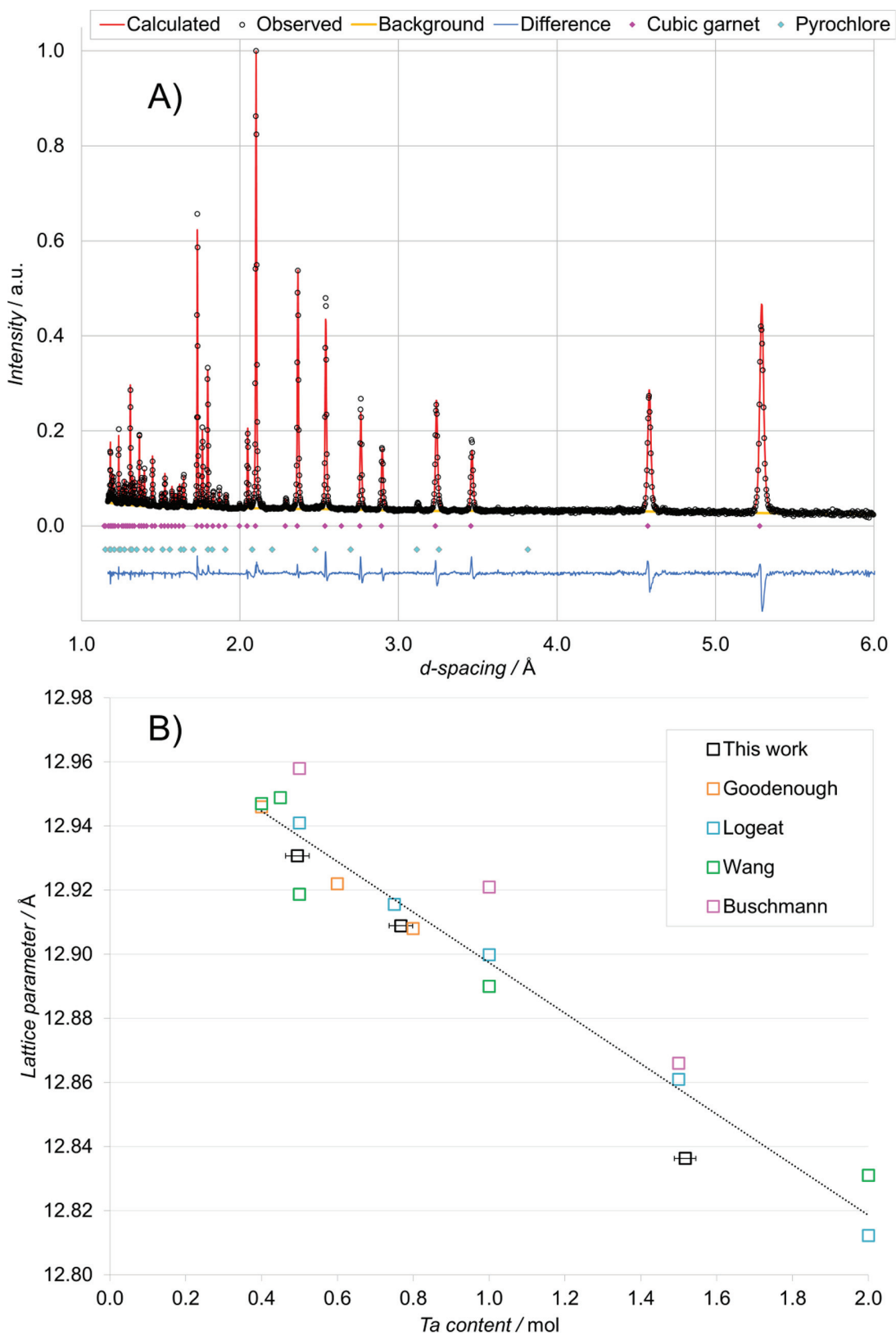

Figure 4. A) Representative plot of refinement of the neutron diffraction data after convergence for the sample with composition of $\mathrm{Li}_{6.5} \mathrm{La}_{3} \mathrm{Zr}_{1.5} \mathrm{Ta}_{0.5} \mathrm{O}_{12}$. B) The lattice parameter versus Ta content for the compositional series compared to other studies available in the literature. ${ }^{[16,34-36}$ The dotted line is a linear fit to all the data on the plot and is included to help guide the eye.

This work proposes that the Li site occupancy is an important factor for defining the effective carrier concentration. Figure 5A,B also plot the Li ionic conductivity on the second $y$-axis. The open circles correspond to the bulk conductivity determined from the equivalent circuit modeling discussed previously while the dotted line is a power fit to total conductivity data of similar Ta-substituted LLZO compositional series available in the literature. ${ }^{[16,17,35,36]}$ It can be seen that the conductivity increases nonlinearly with population of the $96 \mathrm{~h}$ site and depopulation of the $24 \mathrm{~d}$ site. As a result, the increase in conductivity shown in Figure 5A,B cannot be explained by an increase in the Li stoichiometry (i.e., carrier concentration). For example, in this work, the conductivity of $\mathrm{Li}_{6.5} \mathrm{La}_{3} \mathrm{Zr}_{1.5} \mathrm{Ta}_{0.5} \mathrm{O}_{12}$ is $\approx 120 \%$ higher than $\mathrm{Li}$ ${ }_{6.25} \mathrm{La}_{3} \mathrm{Zr}_{1.25} \mathrm{Ta}_{0.75} \mathrm{O}_{12}$ even though the carrier concentration (assuming all the ions participate in conduction) only increases by $4 \%$. Since a wide Li content range was explored with no dopant atoms in the Li sublattice, the transport data (i.e., conductivity increases with an increase in the number of Li-ions in the $96 \mathrm{~h}$ site) corroborate the results of the NMR studies mentioned above. Namely, that the ions occupying the $96 \mathrm{~h}$ site are primarily responsible for the high ionic conductivity observed in Li garnets. If it is assumed that the mobility is constant (as suggested by the work of Ohta et al.), ${ }^{[29]}$ then the conductivity should follow a linear relationship with the carrier concentration. From this work, the carrier concentration was calculated assuming only the ions in octahedral coordination with oxygen (96h) participate in conduction and compared to the bulk conductivity. However, the bulk conductivity still does not change linearly with carrier concentration when this is done. Therefore, the definition of the effective carrier concentration is not all of the Li ions, nor all of the $96 \mathrm{~h}$ ions, but must be further restricted.

To begin the discussion of how the carrier concentration should be defined, this work proposes that the increase in conductivity,

not change enough to explain the increase in conductivity, it was thought that changes to the mobility caused by the dopant must, therefore, be controlling the conductivity. ${ }^{[10]}$ However, recent work by Ohta et al. found that the diffusivity (mobility) does not change with the dopant, and therefore suggested that only a small fraction $(\approx 10 \%-15 \%)$ of the Li-ion ions were acting as carriers. ${ }^{[2]}$ The finding that only a small fraction of the ions may be participating in the conduction is reminiscent of the discussion surrounding other families of ionic conductors. ${ }^{[23-27,48]}$ As a consequence, it is important to discuss how the carriers are defined in the garnet family of ionic conductors. with increasing Li content, is caused by an increase in the effective carrier concentration. It is asserted that the effective carrier concentration does not change proportionally with the $\mathrm{Li}$ stoichiometry. Similar behavior has been observed previously in the literature for $\mathrm{AgPO}_{3}-\mathrm{AgI}$ glasses. ${ }^{[49,50]}$ We propose that the highly nonlinear increase in conductivity as the Li content is increased (Figure 5A,B) is due to a synergistic effect of the Li site distribution on the effective carrier concentration caused by coulombic carrier-carrier interactions. This statement is supported by computational reports in the literature which show significant carrier-carrier interactions in stuffed 
Table 2. Results of the structural refinement for the sample with nominal composition $\mathrm{Li}_{6.5} \mathrm{La}_{3} \mathrm{Zr}_{1.5} \mathrm{Ta}_{0.5} \mathrm{O}_{12}$ at $300 \mathrm{~K}$. Note: Space group la-3d (No. 230); $a=12.9307(4) \AA$; $R_{\mathrm{wp}}=3.19, \chi^{2}=6.31, R_{\mathrm{p}}=5.36$.

\begin{tabular}{|c|c|c|c|c|c|c|}
\hline Name & Site & Site occupancy & $x$ & $Y$ & z & $100 \times \cup_{e q}\left[\AA^{2}\right]$ \\
\hline Li1 & $24 d$ & $0.412(9)$ & $3 / 8$ & 0 & $1 / 4$ & $2.24(15)$ \\
\hline Li2 & $96 \mathrm{~h}$ & $0.375(4)$ & $0.0972(4)$ & $0.6868(4)$ & $0.5790(4)$ & $1.79(11)$ \\
\hline La & $24 c$ & 1.000 & $1 / 8$ & 0 & $1 / 4$ & $1.012(7)$ \\
\hline $\mathrm{Zr}$ & $16 a$ & $0.738(2)$ & 0 & 0 & 0 & $0.806(10)$ \\
\hline $\mathrm{Ta}$ & $16 a$ & $0.247(2)$ & 0 & 0 & 0 & $0.806(10)$ \\
\hline $\mathrm{O}$ & $96 \mathrm{~h}$ & 1.000 & $-0.0317(5)$ & $0.0536(5)$ & $0.1488(5)$ & $1.467(6)$ \\
\hline
\end{tabular}

garnets. ${ }^{[13,14,51,52]}$ Furthermore, as shown in Table 1, comparison of the $\mathrm{Li}-\mathrm{Li}$ bond distances from the results of the structural refinement of this work reveal decreasing 24d-96h and increasing 96h-96h Li-Li separation distances with increasing Li content. Since the previous NMR reports mentioned above show the $24 \mathrm{~d}$ site is less mobile than the $96 \mathrm{~h}$ site, a decreased 24d-96h separation distance would lead to increased coulombic repulsion and destabilization of the ions on the $96 \mathrm{~h}$ site in the Li sublattice. This result is consistent with the observations of highly interactive charge carriers made in computation reports mentioned above. Interestingly, previous work on the AgI family of superionic conductors suggests that highly interacting charge carriers may be a necessary condition for superionic conduction. ${ }^{[48]}$

Carrier-carrier coulombic interactions caused by short LiLi separation distance driven by the change in site occupancy seem to be critical for the high Li-ion conductivity of the garnet family of materials, however, there is a limit to how much destabilization of the Li sublattice can occur. As discussed in the literature previously, as the Li content is increased past 6.5 moles, the system undergoes a reduction of symmetry from the cubic to the tetragonal phase in order to relieve coulombic repulsion in the Li sublattice. ${ }^{[14,15,21]}$ Perhaps the nonlinear increase in the effective carrier concentration, and thus conductivity, could continue to increase as the Li content is increased past $\mathrm{Li}=6.5$ and the $96 \mathrm{~h}$ site is further populated at the expense of the $24 \mathrm{~d}$ site. However, our previous work has shown that phase segregation into a mixture of cubic and tetragonal polymorphs occurs and the net conductivity decreases. ${ }^{[15]}$ Therefore, since the only known approach to change the Li site occupancy is by changing the total Li content via a dopant, we predict that compositions near $\mathrm{Li}=6.5$, where the cubic polymorph is stabilized, represent the compositions with the highest possible bulk conductivity in LLZO. This is because these compositions have the highest occupancy of the $96 \mathrm{~h}$ site, lowest occupancy of the $24 \mathrm{~d}$ site, and, thus, the shortest $24 \mathrm{~d}-96 \mathrm{~h} \mathrm{Li}-\mathrm{Li}$ separation distances while maintaining the cubic phase. Certainly, other factors such as the lattice parameter, degree of covalent bonding in the $\mathrm{MO}_{6}$ structural unit, and the presence of other crystal defects such as oxygen vacancies ${ }^{[53-55]}$ also affect the ionic conductivity. For example, sintering in a pure oxygen atmosphere has been shown to increase the total conductivity of LLZO compared to sintering in air or inert atmosphere. ${ }^{[55]}$ Likewise, isolating the role that defects, such as oxygen vacancies, play in controlling ionic and perhaps electronic conductivity is recommended for future work. Since the introduction of a dopant changes several variables (i.e., Li content, Li site occupancy, lattice parameter, nature of the bonding) and the processing atmosphere can change the defect chemistry, separation of these effects is difficult.

\section{Conclusion}

The garnet materials system simultaneously meets all of the demanding requirements needed in a solid electrolyte for advanced batteries. More than that, though, the garnet materials system could be a new platform for fundamental scientific investigations into the nature of ionic transport in systems with more than one conduction site and significant carrier-carrier interactions. Highly interacting charge carriers are likely responsible for the high Li-ion conductivity observed in the AgI family of materials. This is the first report to show a similar mechanism to that of the AgI family of superionic conductors for the origin of the high ionic conductivity of the garnet family of materials. Certainly more work is needed to fully correlate structural properties (such as site occupancy and Li separation distances) to the degree of coulombic carrier-carrier interaction so that the effective carrier concentration can be defined and quantified. The garnet crystal structure offers unique flexibility to explore a wide compositional space and results can be compared to other solid electrolytes with two conduction sites such as the NaSICON based $\mathrm{Li}_{1+x} \mathrm{Al}_{\mathrm{x}} \mathrm{Ti}_{2-x}\left(\mathrm{PO}_{4}\right)_{3}$ systems. The garnet family of materials could help shed light on the fundamental properties necessary for high solid-state Li-ion conductivity. A better understanding of the variables which control the carrier mobility and concentration can lead to strategies to further increase the ionic conductivity. This work explains why the conductivity increases nonlinearly as the cubic-to-tetragonal phase transition is approached near a $\mathrm{Li}$ content of $\mathrm{Li}=6.5 \mathrm{~mol}$. This work also explains why compositions with just enough Li vacancies to stabilize the cubic phase exhibit the maximum in ionic conductivity. While these types of fundamental questions are being investigated, compositions near $\mathrm{Li}=6.5$ moles should be targeted for development of advanced chemistries at the cell and device level.

\section{Experimental Section}

Samples of composition $\mathrm{Li}_{7-x} \mathrm{La}_{3} \mathrm{Zr}_{2-x} \mathrm{Ta}_{x} \mathrm{O}_{12}(x=0.5,0.75$, and 1.5) were prepared using a coprecipitation process. $\mathrm{Li}_{2} \mathrm{CO}_{3}, \mathrm{La}(\mathrm{OH})_{3}, "\left[\mathrm{ZrO}_{2}\right]_{2}$. $\mathrm{CO}_{2} \cdot \mathrm{xH}_{2} \mathrm{O}$ " (zirconium carbonate, basic hydrate; equivalent $\mathrm{ZrO}_{2}$ content 


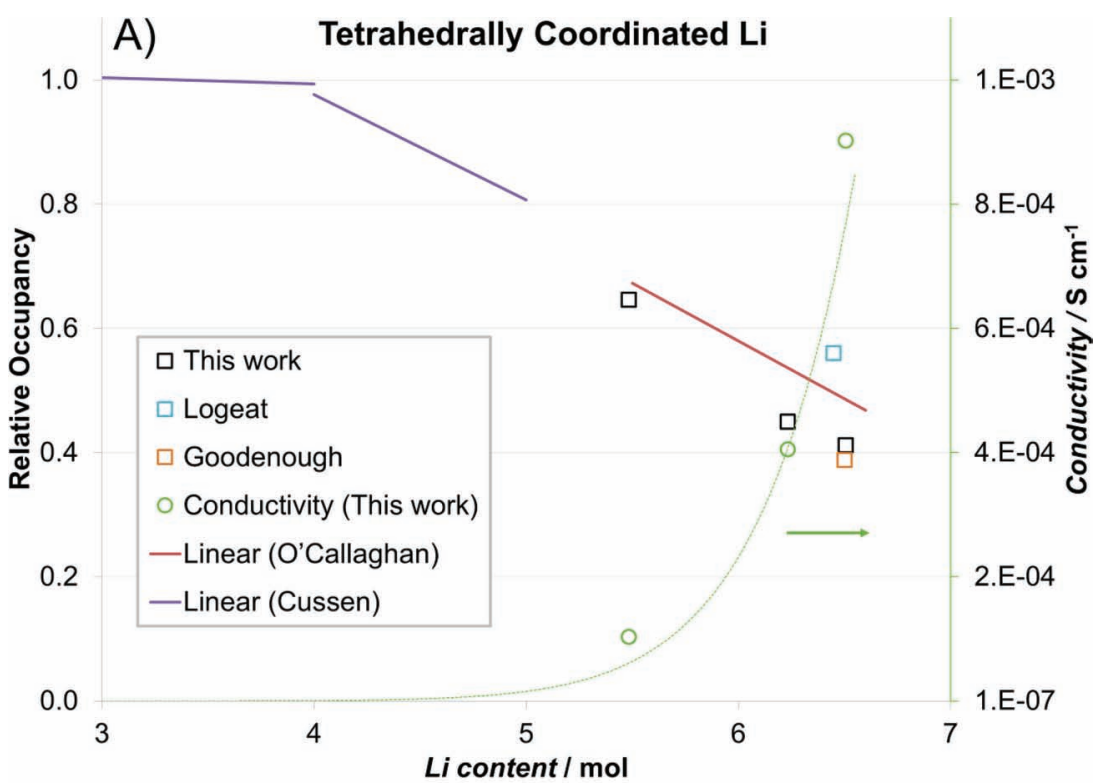

B) Octahedrally Coordinated Li

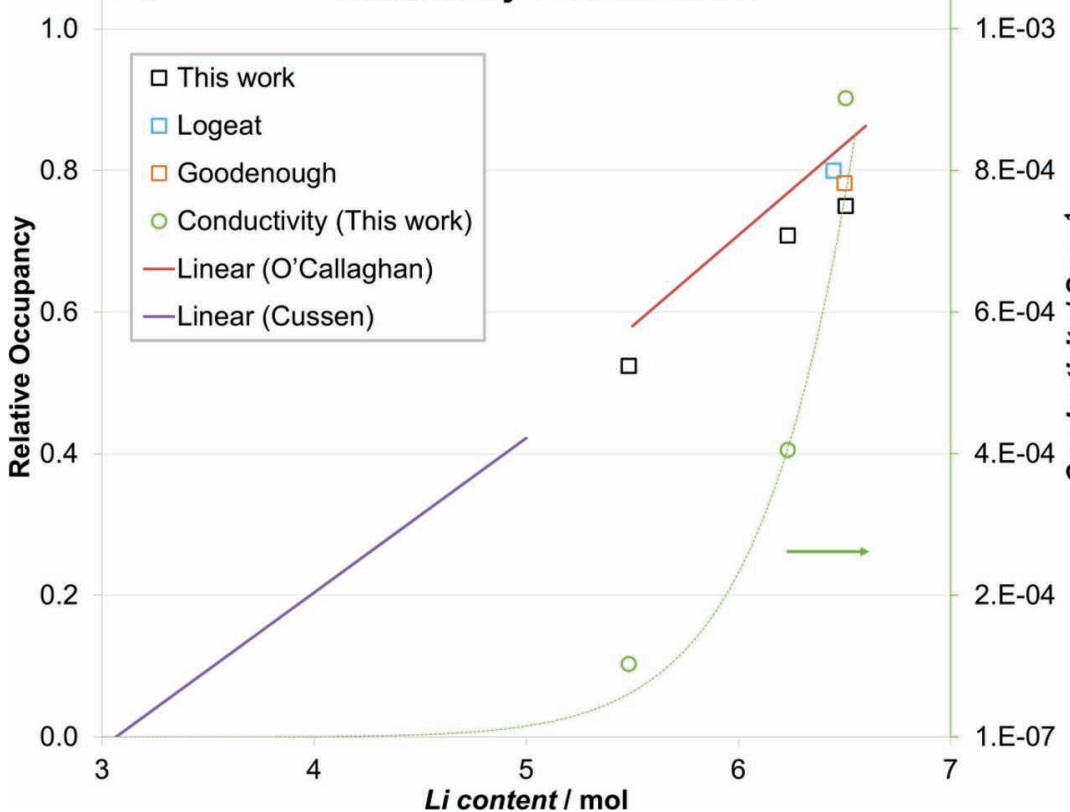

Figure 5. Plot of the tetrahedral A) and octahedral B) Li site occupancies versus Li content comparing the compositional series to the data available in the literature. ${ }^{[16,34,38-42]}$ The ionic conductivity is included and is plotted on the second $y$-axis. For the conductivity data, the open symbols correspond to the bulk conductivity determined from the equivalent circuit modeling while the dotted line is a power fit to total conductivity data of similar Ta-substituted LLZO compositional series available in the literature. ${ }^{[16,17,35,36]}$

determined from thermogravimetric analysis) weighed in the desired stoichiometry were dissolved in $\approx 1.4 \mathrm{M} \mathrm{HNO}_{3}$ (aq). A 6 wt\% excess of $\mathrm{Li}$ was used to compensate for $\mathrm{Li}$ volatilization during synthesis. The stoichiometric amount of $\mathrm{TaCl}_{5}$ was first dissolved in anhydrous ethanol and then added to the $\mathrm{Li}, \mathrm{La}$, and $\mathrm{Zr}$ containing precursor solution. The resulting solution was evaporated to dryness in a microwave oven contained inside a fume hood. Evolution of $\mathrm{NO}_{x}$ was observed during this step. The dried coprecipitate was lightly ground with a mortar and pestle and pressed into a pellet using a Carver laboratory die and press. The pellet was placed on a $\mathrm{ZrO}_{2}$ plate and heated in air at $1173 \mathrm{~K}$ for 3-4 h.
The furnace was turned off and the sample was removed.

The powders were consolidated by rapid induction hot-pressing (RIHP) at $1323 \mathrm{~K}$ and $62 \mathrm{MPa}$ for $1 \mathrm{~h}$ in graphite dies in air under argon shielding gas. The density of each sample was calculated using the bulk geometry and the mass. The density of the sintered samples was compared to what is expected from the crystal structure as determined from Rietveld refinements of the diffraction data.

SEM was performed on a Carl Zeiss Auriga Cross-Beam FIB-FESEM at an accelerating voltage of $15 \mathrm{kV}$ and a working distance of $6.9 \mathrm{~mm} .5 \mathrm{~nm}$ of Pt was sputtered to prevent charging because it is not reactive with $\mathrm{Li}$. The grain size was estimated from the SEM images of the fracture surface using the linear intercept method.

EIS was performed with a Bio-Logic SP200 from 7 $\mathrm{MHz}$ to $10 \mathrm{~Hz}$ with sputtered Pt blocking electrodes. Spectra were collected as a function of temperature from $\approx 289$ to $360 \mathrm{~K}$. A modified equivalent circuit developed by Huggins was used for modeling the data. ${ }^{[33]}$ It should be noted that because of the relatively high frequencies required to capture the full EIS spectrum, not all of the high temperature points could be modeled. It can be seen in Figure 3 that even at room temperature, the characteristic frequency for the bulk response does not occur until $>12 \mathrm{MHz}$ (as estimated by extrapolation of the modeled impedance response to higher frequencies). Thus, the Arrhenius plot of the bulk conductivities (Figure S1, Supporting Information) explicitly excludes points at elevated temperature, which we could not model with certainty. The entire impedance spectra were normalized to the bulk sample geometry before the equivalent circuit modeling was performed. The constant phase elements (CPE) used in the model were observed to behave nearly ideally as is evident by $\alpha$ coefficients refining to values ranging between 0.92 and 1.0. Because of the configuration of the elements in this model, it is impossible to calculate equivalent capacitances for the CPEs. However, since the CPEs exhibited nearly ideal behavior the $Q$ value can be taken as a capacitance as a first approximation. Two conduction processes (i.e., bulk and grain boundary) are observed in the high frequency regime of the Nyquist plot shown in Figure 3. The values of $Q$ for the CPEs for the grain and grain boundary processes are $\approx 10^{-11}$ and $\approx 10^{-8} \mathrm{~F} \mathrm{~cm}^{-2}$ for the bulk and the grain boundaries, respectively, which is in excellent agreement with what is expected. ${ }^{[33,56]}$ The $Q$ value for both the bulk and grain boundary processes did not appreciably change with temperature for all samples.

Time of flight neutron powder diffraction data were collected at the POWGEN beamline at the Spallation Neutron Source (SNS), Oak Ridge National Laboratory (ORNL) with center wavelengths of 1.066 and $2.655 \AA$ at $300 \mathrm{~K}$ to obtain diffraction patterns that spans from $d$-spacing of 0.3-6.5 A. High-resolution synchrotron powder diffraction data were collected using beamline 11-BM at the Advanced Photon Source (APS), Argonne National Laboratory using wavelength of $0.414 \AA$. Rietveld structural refinements of the synchrotron X-ray and neutron diffraction data were performed using GSAS with EXPGUI.[57,58] The synchrotron X-ray source was able to resolve the presence of more 
than one cubic phase where the neutron beamline, let alone lab scale $X$-ray diffractometers, was not. This is significant because the POWGEN beamline is a high-resolution neutron diffraction facility. This is the first observance of multiple cubic LLZO phases coexisting in Ta-doped LLZO but has been observed previously for Al-doped LLZO. ${ }^{[59]}$ Because of this, the $\mathrm{Ta}$ and $\mathrm{Zr}$ concentrations were first determined with the X-ray histogram and a reasonable guess at the Li concentration. The neutron histograms were then treated as a single cubic phase and the refined $\mathrm{Ta}$ and $\mathrm{Zr}$ contents were left fixed so that the Li site distribution could be determined. This process was iterated once where the newly found $\mathrm{Li}$ contents were used to again determine the $\mathrm{Ta}$ and $\mathrm{Zr}$ concentrations. There was no considerable change, the iterations were stopped, and the structure considered solved. Since the weight fraction of each cubic phase was $\approx 50 \%$ and the differences between them were small $(\mathrm{Ta}=0.42$ vs 0.57 mols and $a=13.0134$ vs $12.9529 \AA$, respectively; Ta $=0.74$ vs 0.834 mols and $a=12.9918$ vs $12.9317 \AA$, respectively), the refinement of the diffraction data in this manner was seen as reasonable.

\section{Supporting Information}

Supporting Information is available from the Wiley Online Library or from the author.

\section{Acknowledgements}

T.T., A.S., and J.S. would like to acknowledge support from the Revolutionary Materials for Solid State Energy Conversion, an Energy Frontier Research Center funded by the U.S. Department of Energy, Office of Science, Office of Basic Energy Science under Award No. DE-SC001054. J.W. and J.L.A. would like to acknowledge support of the U.S. Army Research Laboratory (ARL). Funding for M.D.J. was provided by the Office of Naval Research through the Naval Research Laboratory's Basic Research Program. The diffraction research conducted at the Spallation Neutron Source at Oak Ridge National Laboratory and the Advanced Photon Source at Argonne National Laboratory was sponsored by the Scientific User Facilities Division, Office of Basic Energy Sciences, U.S. Department of Energy.

Received: January 13, 2015 Revised: February 16, 2015 Published online: March 21, 2015

[1] J. B. Goodenough, Y. Kim, Chem. Mater. 2010, 22, 587.

[2] M. Armand, J. M. Tarascon, Nature 2008, 451, 652.

[3] R. A. Huggins, Advanced Batteries: Materials Science Aspect, Springer Science and Business Media, New York, NY 2009.

[4] P. G. Bruce, S. A. Freunberger, L. J. Hardwick, J. M. Tarascon, Nat. Mater. 2012, 11, 19.

[5] H. Hyooma, K. Hayashi, Mater. Res. Bull. 1988, 23, 1399.

[6] V. Thangadurai, W. Weppner, Adv. Funct. Mater. 2005, 15, 107.

[7] V. Thangadurai, W. Weppner, lonics 2006, 12, 81.

[8] R. Murugan, V. Thangadurai, W. Weppner, Angew. Chem. Int. Ed. 2007, 46, 7778.

[9] D. T. Wong, S. A. Mullin, V. S. Battaglia, N. P. Balsara, J. Membr. Sci. 2012, 394, 175.

[10] S. Ohta, T. Kobayashi, T. Asaoka, J. Power Sources 2011, 196, 3342.

[11] M. Kotobuki, K. Kanamura, Y. Sato, T. Yoshida, J. Power Sources 2011, 196, 7750.

[12] Y. Shimonishi, A. Toda, T. Zhang, A. Hirano, N. Imanishi, O. Yamamoto, Y. Takeda, Solid State lonics 2011, 183, 48.

[13] M. Xu, M. S. Park, J. M. Lee, T. Y. Kim, Y. S. Park, E. Ma, Phys. Rev. B 2012, 85, 052301.

[14] N. Bernstein, M. D. Johannes, K. Hoang, Phys. Rev. Lett. 2012, 109, 205702.
[15] T. Thompson, J. Wolfenstine, J. L. Allen, M. Johannes, A. Huq, I. N. David, J. Sakamoto, J. Mater. Chem. A 2014, 2, 13431.

[16] Y. Li, J. T. Han, C. A. Wang, H. Xie, J. B. Goodenough, J. Mater. Chem. 2012, 22, 15357.

[17] R. Inada, K. Kusakabe, T. Tanaka, S. Kudo, Y. Sakurai, Solid State Ionics 2013, 262, 568.

[18] A. Gupta, R. Murugan, M. P. Paranthaman, Z. Bi, C. A. Bridges, M. Nakanishi, A. P. Sokolov, K. S. Han, E. W. Hagaman, H. Xie, C. B. Mullins, J. B. Goodenough, J. Power Sources 2012, 209, 184.

[19] S. Ramakumar, L. Satyanarayana, S. V. Manorama, R. Murugan, Phys. Chem. Chem. Phys. 2013, 15, 11327.

[20] K. Saranya, C. Deviannapoorani, L. Dhivya, S. Ramakumar, N. Janani, R. Murugan, Mater. Lett. 2012, 77, 57.

[21] E. Rangasamy, J. Wolfenstine, J. Sakamoto, Solid State Ionics 2012, 206, 28.

[22] J. B. Goodenough, Proc. R. Soc. London 1984, 215, A393.

[23] C. Stuhrmann, H. Kreiterling, K. Funke, Solid State lonics 2002, 154, 109.

[24] K. Funke, R. Hackenberg, Ber. Bunsen-Ges. Phys. Chem. 1972, 76, 883.

[25] W. V. Johnston, H. Wiedersich, G. W. Lindberg, J. Chem. Phys. 1969, 51, 3739.

[26] H. Wiedersich, W. V. Johnston, J. Phys. Chem. Solids 1969, 30, 450.

[27] T. Kaneda, E. Mizuki, Phys. Rev. Lett. 1972, 29, 937.

[28] C. H. J. Stuhrmann, H. Kreiterling, K. Funke, Phys. Chem. Chem. Phys. 2001, 3, 2557.

[29] H. Nozaki, M. Harada, S. Ohta, I. Watanabe, Y. Miyake, Y. Ikedo, N. H. Jalarvo, E. Mamontov, J. Sugiyama, Solid State lonics 2014, 262, 585.

[30] D. Rettenwander, C. A. Geiger, G. Amthauer, Inorg. Chem. 2013, 52, 8005.

[31] D. Rettenwander, P. Blaha, R. Laskowski, K. Schwarz, P. Bottke, M. Wilkening, C. A. Geiger, G. Amthauer, Chem. Mater. 2014, 26, 2617.

[32] J. L. Allen, J. Wolfenstine, E. Rangasamy, J. Sakamoto, J. Power Sources 2012, 206, 315.

[33] R. A. Huggins, lonics 2002, 8, 300.

[34] A. Logéat, T. Köhler, U. Eisele, B. Stiaszny, A. Harzer, M. Tovar, A. Senyshyn, H. Ehrenberg, B. Kozinsky, Solid State lonics 2012, 206, 33.

[35] Y. Wang, W. Lai, Electrochem. Solid-State Lett. 2012, 15, A68.

[36] H. Buschmann, J. Dölle, S. Berendts, A. Kuhn, P. Bottke, M. Wilkening, P. Heitjans, A. Senyshyn, H. Ehrenberg, A. Lotnyk, V. Duppel, L. Kienle, J. Janek, Phys. Chem. Chem. Phys. 2011, 13, 19378.

[37] R. D. Shannon, Acta Crystallogr. 1976, 751, A32.

[38] E. J. Cussen, Chem. Commun. 2006, 4, 412.

[39] E. J. Cussen, T. W. S. Yip, J. Solid State Chem. 2007, 180, 1832.

[40] M. P. O'Callaghan, A. S. Powell, J. J. Titman, G. Z. Chen, E. J. Cussen, Chem. Mater. 2008, 20, 2360.

[41] M. P. O'Callaghan, E. J. Cussen, Chem. Commun. 2007, 20, 2048.

[42] M. P. O'Callaghan, E. J. Cussen, Solid State Sci. 2008, 10, 390.

[43] C. A. Geiger, Elements 2013, 9, 447.

[44] L. van Wullen, T. Echelmeyer, H. Meyer, D. Wilmer, Phys. Chem. Chem. Phys. 2007, 9, 3298.

[45] B. Koch, M. Vogel, Solid State Nucl. Magn. Reson. 2008, 34, 37.

[46] M. P. O'Callaghan, A. S. Powell, J. J. Titman, G. Z. Chen, E. J. Cussen, Chem. Mater. 2008, 20, 2360.

[47] T. L. Spencer, N. Plagos, D. Brouwer, G. R. Goward, Phys. Chem. Chem. Phys. 2014, 16, 2515.

[48] Y. Liou, R. Hudson, S. Wonnell, L. Slifkin, Phys. Rev. B 1990, 41, 10481.

[49] A. Rodrigues, M. Nascimento, C. Bragatto, J. L. Souquet, J. Chem. Phys. 2011, 135, 234504. 
[50] V. Clement, D. Ravaine, C. Déportes, R. Billat, Solid State lonics 1988, 28, 1572.

[51] R. Jalem, Y. Yamamoto, H. Shiiba, M. Nakayama, H. Munakata, T. Kasuga, K. Kanamura, Chem. Mater. 2013, 25, 425.

[52] K. Meier, T. Laino, A. Curioni, J. Phys. Chem. C 2014, 118, 6668.

[53] Y. Kihira, S. Ohta, H. Imagawa, T. Asaoka, ECS Electrochem. Lett. 2013, 2, A56.

[54] H. Xie, Y. Li, J. Han, Y. Dong, M. P. Paranthaman, L. Wang, M. Xu, A. Gupta, Z. Bi, C. A. Bridges, M. Nakanishi, A. P. Sokolov, J. B. Goodenough, J. Electrochem. Soc. 2012, 159, A1148.
[55] Y. Li, Z. Wang, C. Li, Y. Cao, X. Guo, J. Power Sources 2014, 248, 642.

[56] J. T. S. Irvine, D. C. Sinclair, A. R. West, Adv. Mater. 1990, 2, 132.

[57] A. C. Larson, R. B. Von Dreele, General Structure Analysis System (GSAS), Report LAUR 86-748, Los Alamos National Laboratory, Los Alamos, NM 2000.

[58] B. H. Toby, J. Appl. Crystallogr. 2001, 34, 210.

[59] A. A. Hubaud, D. J. Schroeder, B. Key, B. J. Ingram, F. Dogan, J. T. Vaughey, J. Mater. Chem. A 2013, 1, 8813. 